\title{
Vida Fisicamente Ativa como Medida de Enfrentamento ao COVID-19
}

\section{Physically Active Lifestyle as an Approach to Confronting COVID-19}

\author{
Maycon Junior Ferreira, ${ }^{7}$ Maria Cláudia Irigoyen, ${ }^{2}$ Fernanda Consolim-Colombo, ${ }^{2,3}$ José Francisco Kerr Saraiva, ${ }^{4}$ \\ Kátia De Angelis 1,3 (1) \\ Laboratório de Fisiologia do Exercício - Departamento de Fisiologia - Universidade Federal de São Paulo (UNIFESP), ${ }^{1}$ São Paulo, SP - Brasil \\ Instituto do Coração (InCor) - Faculdade de Medicina - Universidade de São Paulo, ${ }^{2}$ São Paulo, SP - Brasil \\ Universidade Nove de Julho (UNINOVE), ${ }^{3}$ São Paulo, SP - Brasil \\ Pontifícia Universidade Católica de Campinas (PUC-Campinas), ${ }^{4}$ Campinas, SP - Brasil
}

A rápida e incontrolável disseminação do novo coronavírus (SARS-CoV-2) pelo mundo aliada à sua gravidade fez com que, no dia 11 de março de 2020, o Diretor-Geral da Organização Mundial da Saúde (OMS) caracterizasse a situação como pandemia. ${ }^{1}$ Atualmente, nota-se um esforço cada vez mais intenso por parte das organizações de saúde e do poder público no sentido de conter o avanço e disseminação do SARS-CoV-2. O SARS-CoV-2 emerge como um novo subtipo de síndrome respiratória aguda grave (SARS-CoV) humana caracterizado por alta capacidade de transmissão e indução de quadros de infecção respiratória severa. Estudos recentes demonstraram alta prevalência de hipertensão arterial e diabetes em pacientes idosos acometidos pela COVID-19 que vieram a óbito em Wuhan, China, ${ }^{2,3}$ epicentro do surto de SARS-CoV-2, o que sugere que tais comorbidades são importantes fatores de risco para o agravamento e pior prognóstico das complicações associadas ao COVID-19.

Diante das recomendações de isolamento social atualmente impostas em vários países, incentivar a manutenção de uma rotina de vida fisicamente ativa por parte da população como uma medida preventiva para a saúde é fundamental durante esse período de enfrentamento contra a disseminação do vírus. Em um período de reclusão domiciliar a população tende a adotar uma rotina sedentária, o que favoreceria a um aumento no ganho de peso corporal e surgimento de comorbidades associadas a maior risco cardiovascular, como obesidade, aumento da pressão arterial, intolerância à glicose, bem como transtornos psicossociais como ansiedade e depressão. De fato, o comportamento sedentário, seja sentado, assistindo TV ou passando tempo em frente a dispositivos eletrônicos, foi associado a aumento do peso corporal em crianças, ${ }^{4}$ adolescentes, ${ }^{5}$ adultos e idosos ${ }^{6}$ e aumento marcante do risco de mortalidade cardiovascular. ${ }^{7}$ Por outro lado, o risco de desenvolvimento de doenças cardiovasculares e mortalidade mostra-se reduzido em indivíduos com hábitos de vida fisicamente ativos, como caminhadas de moderada

\section{Palavras-chave}

Coronavirus-19; COVID-19; Exercício; Atividade Motora; Sedentarismo; Fatores de Risco; Prevenção e Controle.

Correspondência: Kátia De Angelis •

Universidade Federal de São Paulo (UNIFESP), Edifício de Ciências

Biomédicas, Departamento de Fisiologia (5o andar) - Rua Botucatu, 862.

CEP 04023901, Vila Clementino, São Paulo, SP - Brasil

E-mail: prof.kangelis@yahoo.com.br

DOI: https://doi.org/10.36660/abc.20200235 intensidade. ${ }^{8}$ Além disto, tem sido demonstrado que o risco de infecção do trato respiratório superior por coronavírus é potencialmente maior na presença de deficiência do sistema imunológico. ${ }^{9}$ Neste sentido, a prática de exercício físico como medida benéfica para a melhora da imunidade é fortemente evidenciada na literatura. ${ }^{10-12} \mathrm{O}$ Colégio Americano de Medicina do Esporte divulgou recentemente um guia em que sugere que a atividade física de intensidade moderada deva ser mantida no período de quarentena em função do SARS-CoV-2, salientando a importância para a saúde de cada minuto fisicamente ativo. ${ }^{13}$

Vale lembrar que as recomendações da OMS para indivíduos saudáveis e assintomáticos são de, no mínimo, 150 minutos de atividade física por semana para adultos e 300 minutos de atividade física por semana para crianças e adolescentes. ${ }^{14}$ Esse tempo de atividade física deve ser acumulado durante os dias da semana, podendo ser dividido de acordo com a rotina do sujeito, sendo composto preferencialmente por atividades físicas de intensidade moderada e intensa. Ressaltamos a importância da orientação de profissionais do exercício físico para a adequação da prática de atividade física por parte da população. É fundamental que indivíduos que realizem exercícios físicos regularmente mantenham a prática, porém adequando-se à condição atual de restrições de circulação. Manter-se fisicamente ativo deve ser enfatizado ainda mais para indivíduos idosos, os quais comprovadamente apresentam mais comorbidades e maior risco cardiovascular, além de serem mais vulneráveis ao COVID-19. Populações com comorbidades cardiovasculares devem realizar atividades físicas diariamente, mantendo o tratamento farmacológico e respeitando suas eventuais limitações físicas e as recomendações de profissionais de saúde. A prática de exercícios físicos deve ser interrompida na presença de sintomas relacionados ao COVID-19 como febre, tosse seca e dispneia, quando em repouso.

É importante destacar que o ambiente domiciliar e familiar também é propício para realização de atividade física. Desta forma, independentemente da faixa etária, devem ser recomendados à população alguns comportamentos e atitudes que ajudarão na manutenção de uma vida fisicamente ativa, da saúde física e mental e serão importantes para o enfrentamento deste momento de isolamento social, tais como:

- realizar atividades físicas que sejam prazerosas, explorando espaços domiciliares e utensílios disponíveis para se movimentar;

- realizar atividades de vida diária como limpeza, manutenção e organização dos espaços domésticos; 
- brincar e se exercitar com as crianças, adolescentes e animais de estimação, resgatando brincadeiras e jogos que promovam gasto energético superior à condição de repouso;

- evitar o comportamento sedentário, intercalando o tempo sentado ou deitado com períodos de atividade física, reduzindo o tempo de uso de dispositivos eletrônicos;

- reservar alguns minutos para atividades de alongamento, relaxamento e meditação.

\section{Referências}

1. World Health Organization. (WHO) WHO Director-General's opening remarks at the media briefing on COVID-19. [Internet] [Cited in 2020 Mar22] Available from: https://www.who.int/dg/speeches/detail/whodirector-general-s-opening-remarks-at-the-media-briefing-on-covid-19-11-march-2020.

2. Zhou F, Yu T, Du R, Fan G, Liu Y, Liu Z, et al. Clinical course and risk factors for mortality of adult inpatients with COVID-19 in Wuhan, China: a retrospective cohort study. Lancet.2020 Mar 11.pii:S0140-6736(20)30566-3

3. Wu C, Chen X, Cai Y, Xia J, Zhou X, Xu S, et al. Risk factors associated with acute respiratory distress syndrome and death in patients with coronavirus disease 2019 pneumonia in Wuhan, China. JAMA Intern Med. 2020 Mar 13 [Epub ahead print]

4. Tanaka C, Reilly JJ, Tanaka M, Tanaka S. Changes in weight, sedentary behaviour and physical activity during the school year and summer vacation. Int J Environ Res Public Health. 2018 May 4 ;15(5)pii:E915.

5. Cureau F V, Sparrenberger K, Bloch K V, Ekelund U, Schaan BD. Associations of multiple unhealthy lifestyle behaviors with overweight/obesity and abdominal obesity among Brazilian adolescents: a country-wide survey. Nutr Metab Cardiovasc Dis. 2018;28(7):765-74.

6. Banks E, Jorm L, Rogers K, Clements M, Bauman A. Screen-time, obesity, ageing and disability: findings from 91266 participants in the 45 and up study. Public Health Nutr. 2011;14(1):34-43.
Desta forma, diante do avanço exponencial desta pandemia no Brasil, a recomendação dos profissionais de saúde para que a população tenha uma vida fisicamente ativa deve ser encarada como uma importante abordagem para o combate ao COVID-19 e às eventuais consequências do confinamento social juntamente às demais medidas que estão sendo adotadas pelos setores de saúde pública mundial.

7. Patterson R, McNamara E, Tainio M, de Sá TH, Smith AD, Sharp SJ, et al. Sedentary behaviour and risk of all-cause, cardiovascular and cancer mortality, and incident type 2 diabetes: a systematic review and dose response meta-analysis. Eur J Epidemiol. 2018;33(9):811-29.

8. Hamer M, Chida Y. Walking and primary prevention: a meta-analysis of prospective cohort studies. Br J Sports Med. 2008;42(4):238-43.

9. Channappanavar R, Perlman S. Pathogenic human coronavirus infections: causes and consequences of cytokine storm and immunopathology. Semin Immunopathol. 2017;39(5):529-39.

10. Gleeson M, Bishop NC, Stensel DJ, Lindley MR, Mastana SS, Nimmo MA. The anti-inflammatory effects of exercise: mechanisms and implications for the prevention and treatment of disease. Nat Rev Immunol. 2011;11(9):607-15.

11. Suzuki K. Chronic inflammation as an immunological abnormality and effectiveness of exercise. Biomolecules. 2019 Jun 7 ;9(6).pii: E223

12. Pedersen BK. Anti-inflammatory effects of exercise: role in diabetes and cardiovascular disease. Eur J Clin Invest. 2017;47(8):600-11.

13. American College of Sports. (ACSM). Staying active during the coronavirus pandemic. [Internet]. [Cited in 2020 Mar 16] Available from: https://www. exerciseismedicine.org/assets/page documents/EIM Rx\%20for\%20 Health_\%20Staying\%20Active\%20During\%20Coronavirus\%20Pandemic.pdf

14. World Health Organization. (WHO) . Global recommendations on physica activity for health. Geneva; 2010. 